CERN-PPE/96-174

24 September 1996

\title{
BIDIMENSIONAL FOCUSING: A NEW APPROACH TO ATOMIC BEAM TARGETS
}

\author{
L. Dick ${ }^{1) 2)}$, S. Spagocci' ${ }^{2)}$ 3) W. Kubischta \\ CERN, Geneva, Switzerland
}

\begin{abstract}
A new optics for the production of a polarized atomic beam target is described here, allowing to reach an estimated target thickness of $\sim 3 \cdot 10^{13}$ atoms $\mathrm{cm}^{-2}$, for a $10 \mathrm{~cm}$ target length. The system, composed of two orthogonal and decoupled magnet arrays, can be built in a rather open geometry and gives higher target thickness and greater design flexibility as compared to the conventional axially symmetric optics.
\end{abstract}

Submitted to Nuclear Instruments and Methods for Physics Research

\footnotetext{
1) To whom all correspondence should be addressed.

2) Also at Physics Department, Milan University, Italy.

3) Present address:University College London - Department of Physics \& Astronomy
} 
In the conventional approach [1] the atomic hydrogen beam is created by dissociation of molecular hydrogen and passage through a convergent cooled $(30-80 \mathrm{~K})$ nozzle and a skimmer. The beam enters an array of axially symmetric sextupoles, where two of the four hyperfine states are focused through Stern-Gerlach filtering (the atoms feel a force proportional to the product of their magnetic moment and the field gradient). Half of the atoms are lost in the process, partially hitting the magnet walls. RF transition units then provide proton spin polarization.

Let us define as the "jet target thickness" the number of atoms per square centimeter that the accelerator beam encounters while crossing the jet target. In order to increase jet target thickness, perpendicularly to the accelerator beam axis the target should have roughly the same width as the beam (typically a few millimeters), while the constraints on target length along the beam axis are usually less severe.

Apart from aberrations, at the end of a linear focusing system such as a sextupole, one has an image of the nozzle. Therefore, increasing jet target thickness requires a rectangular nozzle with a narrow side (typically less than one millimeter, taking aberrations into account). The other side, however, has to be quite long (typically a few centimeters), in order to keep the density in the dissociator below the critical level $\left(\sim 5 \cdot 10^{16}\right.$ atoms $\mathrm{cm}^{-3}$ [2]), beyond which volume recombination would become important. The longer side of the nozzle has to be placed parallel to the accelerator beam.

The less severe constraints on jet length along the beam direction suggest that cylindrically symmetric devices, like sextupoles, might not be the best choice. Such devices cannot be well matched to a long and narrow nozzle; off-axis acceptance, in fact, is significantly lower. Moreover, they tend to produce an image of the nozzle for a rather narrow velocity region, while the other velocities form a broad halo around the central peak. They also have a more closed geometry, therefore trapping more atoms lost for beam collisions.

\section{$2 \quad$ Bidimensional focusing}

In order to overcome those disadvantages, we were led to consider "bidimensional" magnets [3] (magnets having a certain cross section in a plane, and translational symmetry orthogonally to that plane). In this choice, we were guided by the analogy with cylindrical lenses in optics. Bidimensional magnets can only be approximated in the real world. Practically, magnet depth along the axis of translational symmetry has to be longer than the jet envelope of a quantity of the order of one gap (distance between the poles).

Forces in such magnets lie orthogonally to the midplane, as opposed to axially symmetric systems, where forces are radial. Therefore, they give drift motion along their axis of translational symmetry, while they focus in each plane orthogonal to it. In order to image one of the nozzle's sides one has then to put the other side orthogonally to the family of focusing planes. Two orthogonal arrays are used, each one taking care of a given side.

The first system, acting in a plane orthogonal to the long side of the nozzle, gives an image of the short side, typically measuring a few millimeters. It consists of an array of magnetic lenses (bidimensional magnets dominated by the sextupole component).

The second system parallelizes trajectories, in order to control target size along the accelerator beam axis. Since it would not be possible to use magnetic lenses in the non-imaging plane, due to the length of the nozzle and the need to decouple the two arrays, we decided to use magnetic mirrors. Magnetic mirrors [4] are arrays of poles of alternating sign. They reflect atoms hitting them if they fall within a critical angle $\vartheta$ to the surface [4].

In the following, magnets of the first kind will be called "imaging magnets" and magnets of the 
second kind "non-imaging magnets".

Imaging and non-imaging magnets are whenever possible alternated, in order to decouple the two systems and realize a rather open geometry, for better pumping of residual gas. Choosing a suitable wavelength, however, it is possible to reduce coupling problems to a negligible level, should the two sections coexist in a frame. The mirror field, in fact, falls exponentially away from the pole-tips, with a decay length of the order of one wavelength [4] (the minimum distance between the centers of two poles of equal sign).

With the magnet layout just described, acceptance is nearly uniform along the nozzle, even when long and narrow. The system can be designed in order to give a good image over a broad velocity region and the geometry is much more open.

Although some preliminary indications had been obtained by S.Spagocci in his thesis work [3], a possible way to build such magnets was suggested by L.Dick, who proposed to use the gradient properties of permanent magnet wigglers. ${ }^{2)}$ First introduced by K.Halbach [6] for charged particles, permanent magnet wigglers are arrays of equally wide blocks, such that the magnetization direction rotates by $\frac{2 \pi}{M}$ from one block to the nearest neighbour (see fig.1). One usually chooses $M=4$. Blocks are made up of current sheet equivalent materials, like rare earth-cobalt alloys [6]. Those materials have an almost linear demagnetization curve and behave like a vacuum with a superimposed magnetic charge density given by $\mathbf{B}_{\mathbf{r}} \cdot \mathbf{n}$, where $B_{r}$ is the remanent field (up to $1.38 \mathrm{~T}$ [7]) and $\mathbf{n}$ is the normal to the magnet surface. To a good approximation, the superposition principle applies, making such magnets easy to treat analytically.

\section{$3 \quad$ Focusing properties of Halbach wigglers}

A formula for the Halbach magnet field is given in ref. [6]; from this formula we were able to study off-axis behaviour and derive some simple expressions for its sextupole and quadrupole components [8]. The results may be summarized by saying that the off-axis field is a sort of hybrid between sextupole and quadrupole fields, plus higher order components.

Numerical simulations and analytical considerations led us to the following rule of thumb:

"The minimum focal length is obtained with 1 gap $\leq \lambda \leq 2$ gaps. $\lambda \approx 1$ gap gives a sextupole field with higher order contributions at the pole-tips, helping to bend high velocity atoms. $\lambda \approx$ 1.5 gaps gives a good sextupole field. $\lambda \approx 2$ gaps gives a sextupole field with some quadrupole contributions (that is some spherical aberrations). $\lambda \leq 0.5$ gaps gives a mirror field".

In fig. 2 we show field lines for these cases; it is interesting to note that the field is almost totally confined into the region between the magnet's poles.

A simple formula [8] was found for the focal length of a cell, from which the above mentioned results on magnet multipolarities could be derived, and found to agree with numerical simulations. The focal length was found to depend on $v^{2}$, as it should.

In ref. [3] formulas for the field of a uniformly magnetized current sheet equivalent material block were given. Using such formulas, quadrupole and sextupole components were calculated for an isolated Halbach wiggler cell, and found to agree within a few percent with values for the analogous component, as calculated with Halbach's formula [8]. This shows that each block acts almost independently of the others and Halbach's formula, which strictly refers to an infinite array, can be used to a good approximation even for a few cell blocks.

Typical pole-tip fields obtained are $B \approx 1 T$; for a typical velocity $v \approx 1000 \mathrm{~m} / \mathrm{s}$ we get a sextupole acceptance of $\sim 0.1 \mathrm{rad}[1]$ and a mirror critical angle of the same order [4] .

2) The distinction between "wigglers" and "undulators" is only relevant to synchrotron radiation production [5], so we will loosely use the term "wiggler" everywhere in the article. 


\section{$4 \quad$ Magnet layout}

In fig. 3 and fig. 4 we show the best layout found up to now for a $10 \mathrm{~cm}$ target length and $1.38 T$ remanent field.

For the imaging section, we chose $\lambda=1 \mathrm{gap}$. With this choice, spherical aberrations near the magnet axis are kept at an acceptable level and one has a mirror effect at the pole-tips, helping to bend high velocity tail atoms (low velocity tail atoms are bent through second order focusing). For the non-imaging section, we chose $\lambda=1 \mathrm{~cm}$ (magnetic mirror regime). Nozzle dimensions were chosen to be $40 \times 0.4 \mathrm{~mm}$; it has then the same area as a $4.5 \mathrm{~mm}$ diameter round nozzle.

\section{$5 \quad$ Simulations and results}

In our simulations, based on a ray-tracing program used to design the superconducting sextupoles of the HELP project [9], we assumed a supersonic maxwellian distribution function (a maxwellian distribution with a superimposed drift velocity) [10]. Distribution parameters were given the following values: most probable velocity $=1100 \mathrm{~m} / \mathrm{s}, \mathrm{FWHM}=600 \mathrm{~m} / \mathrm{s}$ (typical values for $30-50 \mathrm{~K}$ nozzle temperature [11]). The resulting Mach number was 2.8. For the $\vartheta$ angle distribution we chose [12]: $f(\vartheta)=6 \sin \vartheta \cos ^{5} \vartheta$. The $\varphi$ angle and nozzle coordinate distributions were assumed to be uniform.

In fig. 3 and fig. 4 we show trajectories for the most probable velocity. In fig.5 and fig.6 we show target thickness profiles for the imaging and non-imaging sections. In the imaging plane one can notice a sharp central peak of $\sim 3 \mathrm{~mm}$ FWHM. In fig.7 we show the acceptance vs velocity diagram.

Target thickness profiles were folded with the accelerator beam profile, assumed to be gaussian with $\sigma=1 \mathrm{~mm}$. For 1 atom $/ \mathrm{sec}$ flux we obtained:

$$
T T=4.8 \cdot 10^{-7} \text { atoms } / \mathrm{cm}^{2}(10 \mathrm{~cm} \text { target })
$$

A run made with flipped electron spin gave $T T=4.4 \cdot 10^{-10}$ atoms $/ \mathrm{cm}^{2}$, implying a nearly perfect rejection efficiency.

Assuming operation at $4 \mathrm{mbarl} / \mathrm{s}$, with $80 \%$ dissociation and $70 \%$ trasmission factor for beam scattering (an educated guess from [11] , [13] and [14] ), it was estimated that:

$$
T T=2.7 \cdot 10^{13} \text { atoms } / \mathrm{cm}^{2}(10 \mathrm{~cm} \text { target })
$$

Such results might be improved, as optimization studies are still being carried out. 


\section{References}

[1] W.Kubischta in Proceedings of the Workshop on Polarized Targets for Storage Rings, Heidelberg, 1991, p. 34-41

[2] D.Singy et al., Nucl. Instr. and Meth., B47 (1990) 167

[3] S.Spagocci, Laurea Thesis, Milan University (1993) (supervised by L.Dick)

[4] G.Brautti, Nucl. Instr. and Meth., 116 (1974) 609

[5] G.Brown,K.Halbach,J.Harris,H.Winick, Nucl. Instr. and Meth., 208 (1983) 65

[6] K.Halbach, Nucl. Instr. and Meth., 187 (1981) 109

[7] Vacuumschmelze GMBH, private communication

[8] L.Dick,S.Spagocci, to be published

[9] W.Kubischta in HELP Collaboration, A Proposal for an Internal Jet-Target Experiment at LEP, CERN/LEPC 93-14 LEPC/P7 (1993) and its Addendum, CERN/LEPC 94-1 LEPC/P7 Add.1

[10] D.R.Miller in Atomic and Molecular Beam Methods, Oxford University Press, New York - Oxford, 1988, G.Scoles ed., p. 14-53

[11] G.Cinque, Doctorate Thesis, Ferrara University (1994)

[12] L.Dick,W.Kubischta, CERN-EP 88-135 (1988)

[13] E.Bosisio, Laurea Thesis, Milan University (1991)

[14] G.Boero,W.Kubischta, CERN/HELP note, 47 (1994) 
Fig.1 A Halbach wiggler with $M=4$

Fig.2 Field lines for the cases: $\lambda=2$ gaps, $\lambda=1.5$ gaps, $\lambda=1$ gap, $\lambda=0.5$ gaps

Fig.3 Atom trajectories for $v=1100 \mathrm{~m} / \mathrm{s}$ (imaging section)

Fig.4 Atom trajectories for $v=1100 \mathrm{~m} / \mathrm{s}$ (non-imaging section)

Fig.5 Target thickness profile (imaging section)

Fig.6 Target thickness profile (non-imaging section)

Fig.7 Acceptance vs velocity diagram 

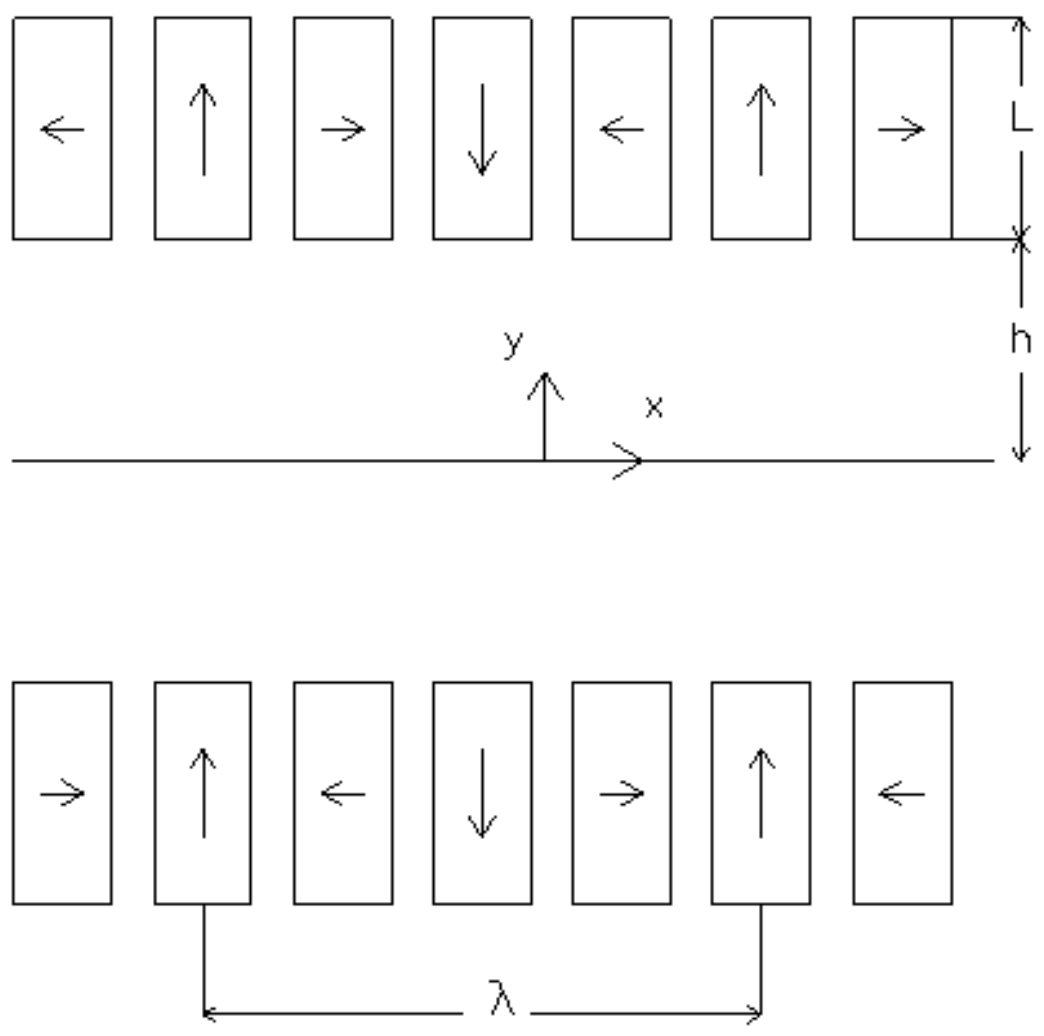

Figure 1: 

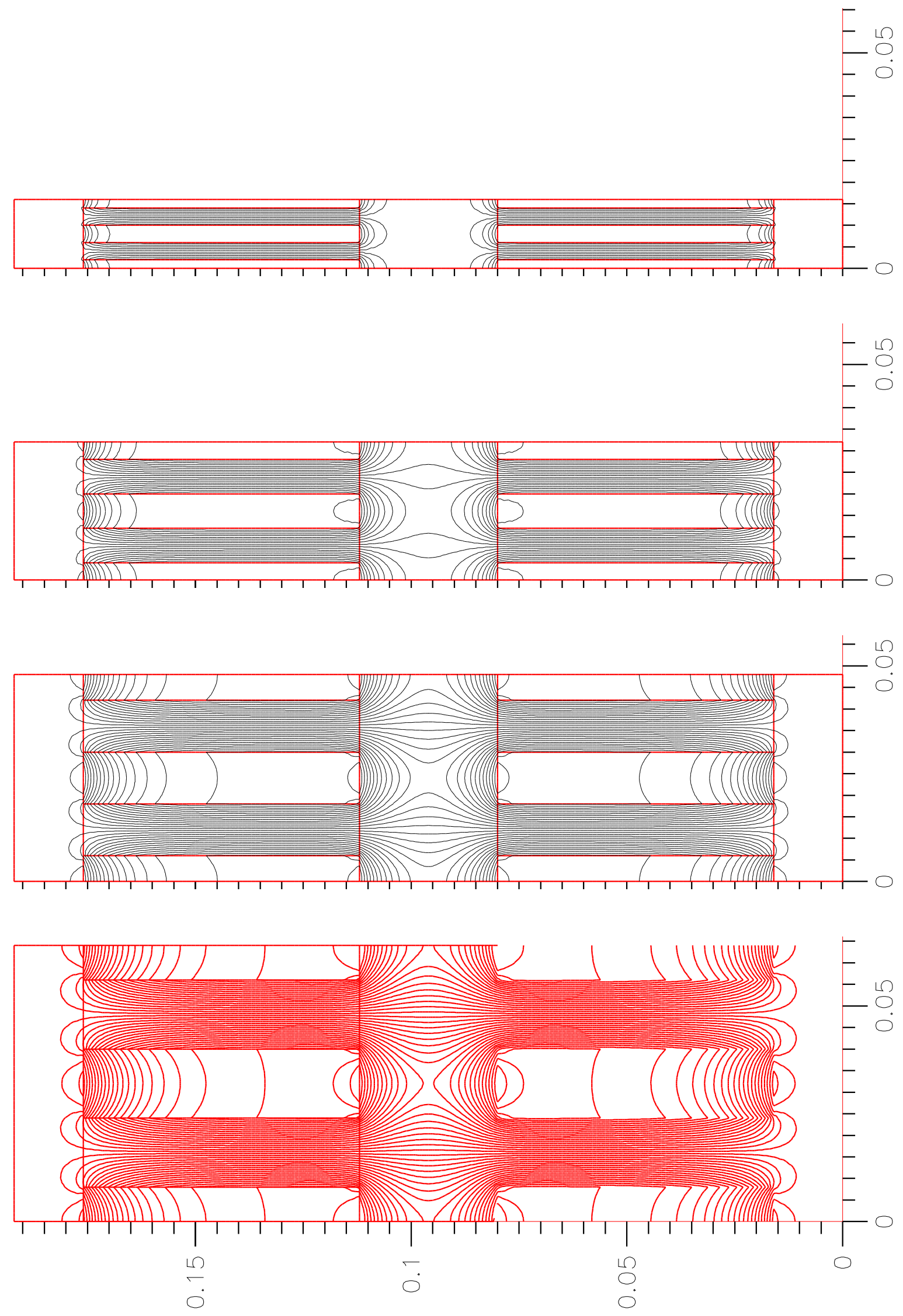

Figure 2: 


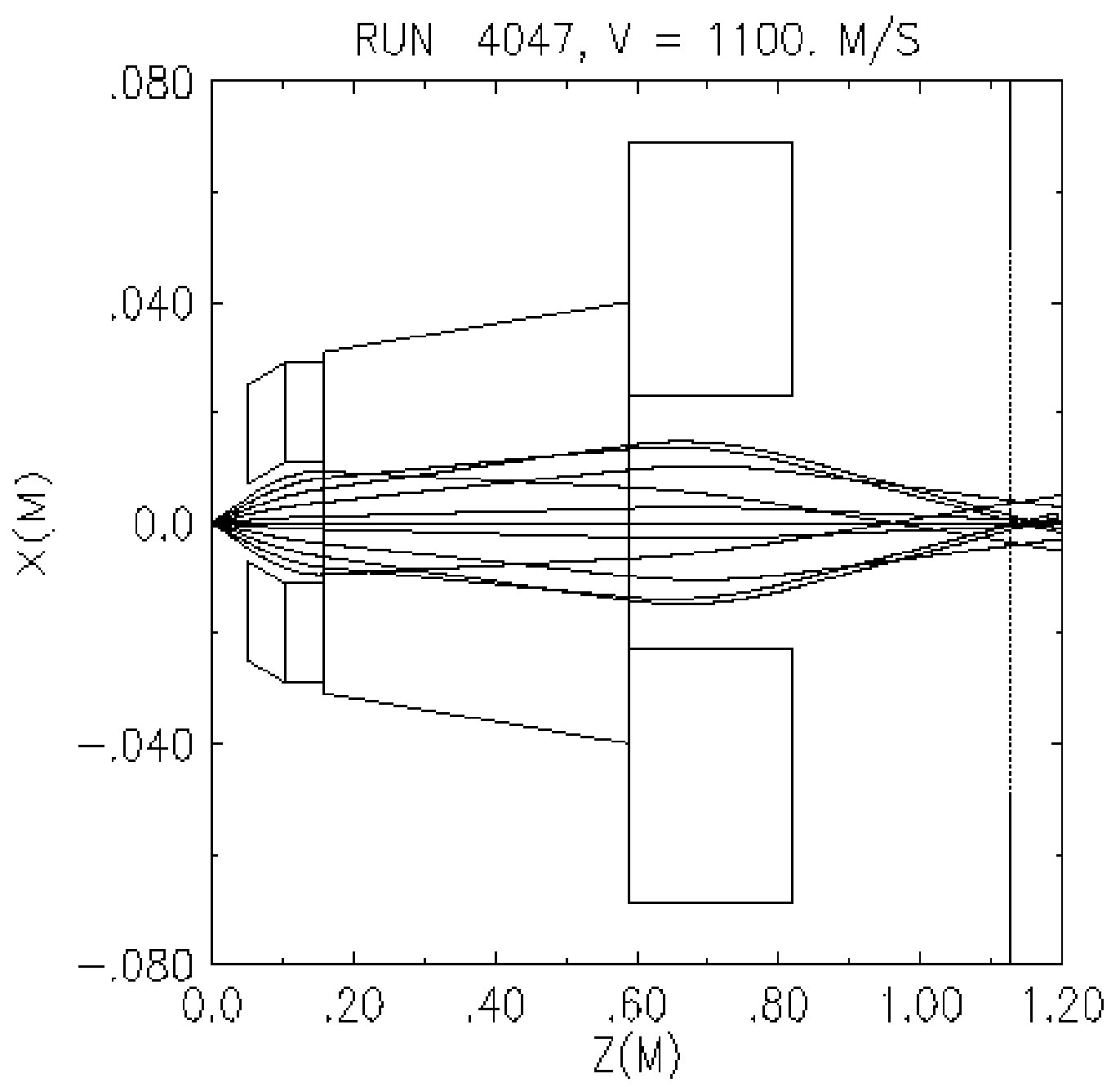

Figure 3: 


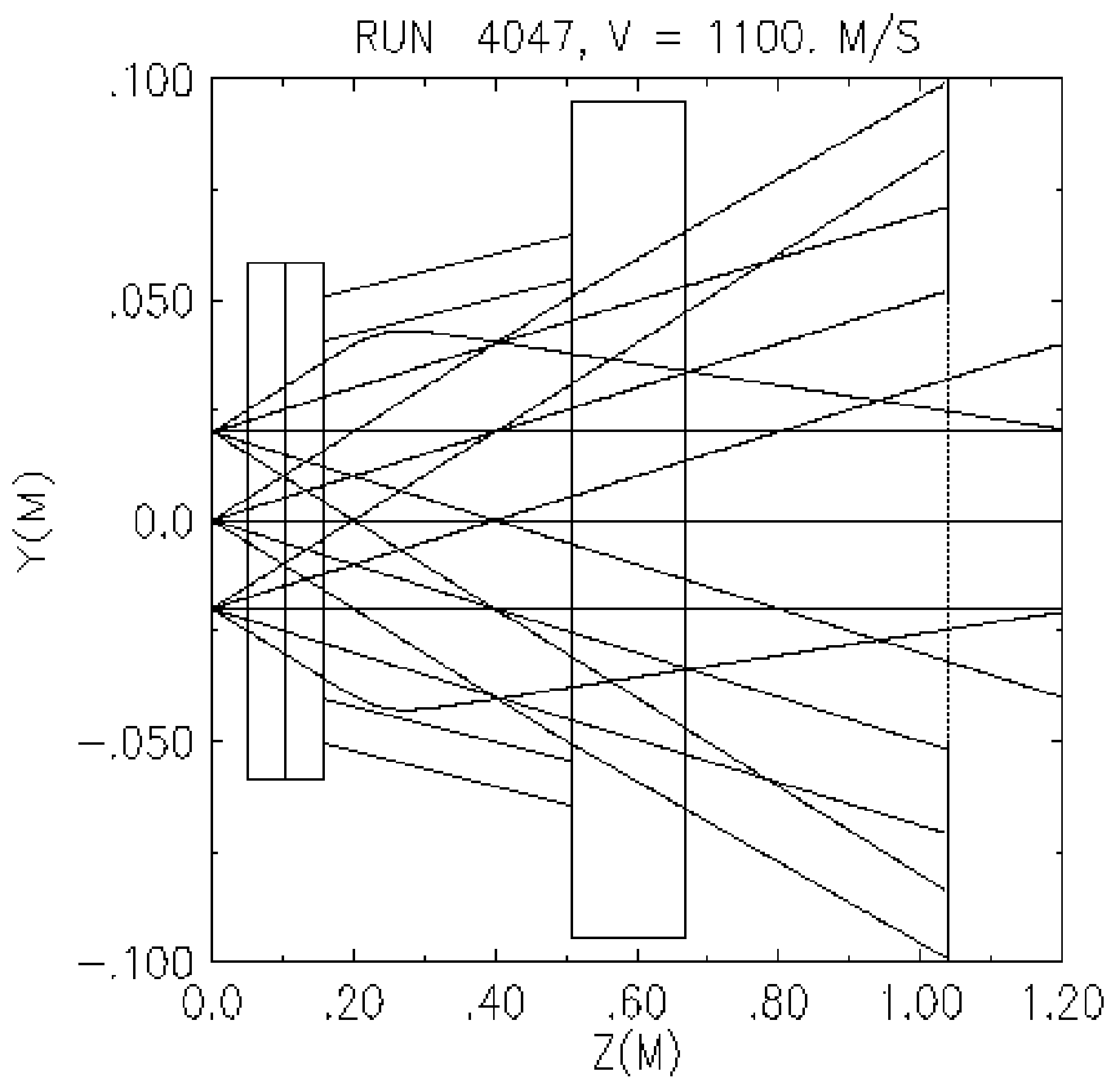

Figure 4: 


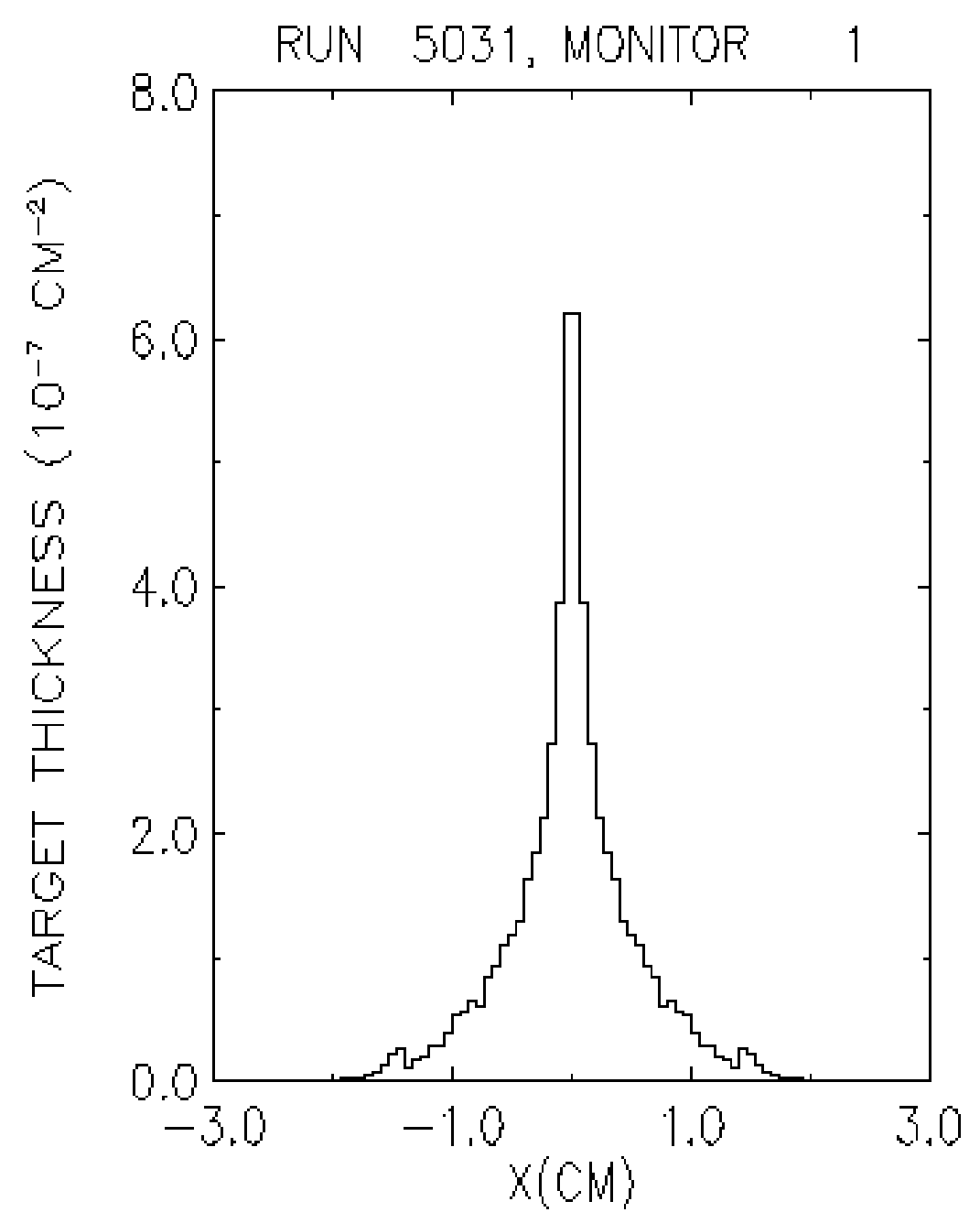

Figure 5: 


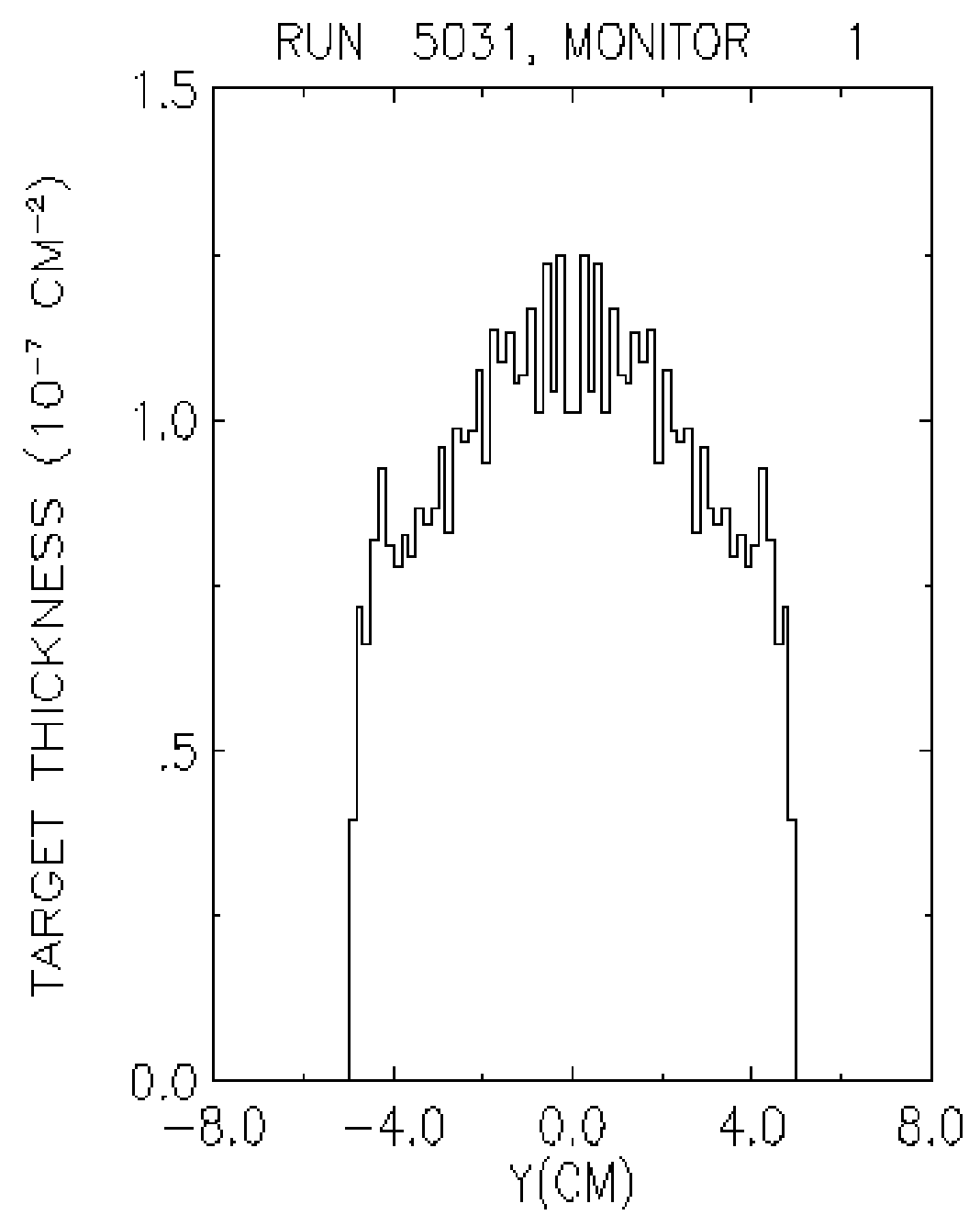

Figure 6: 


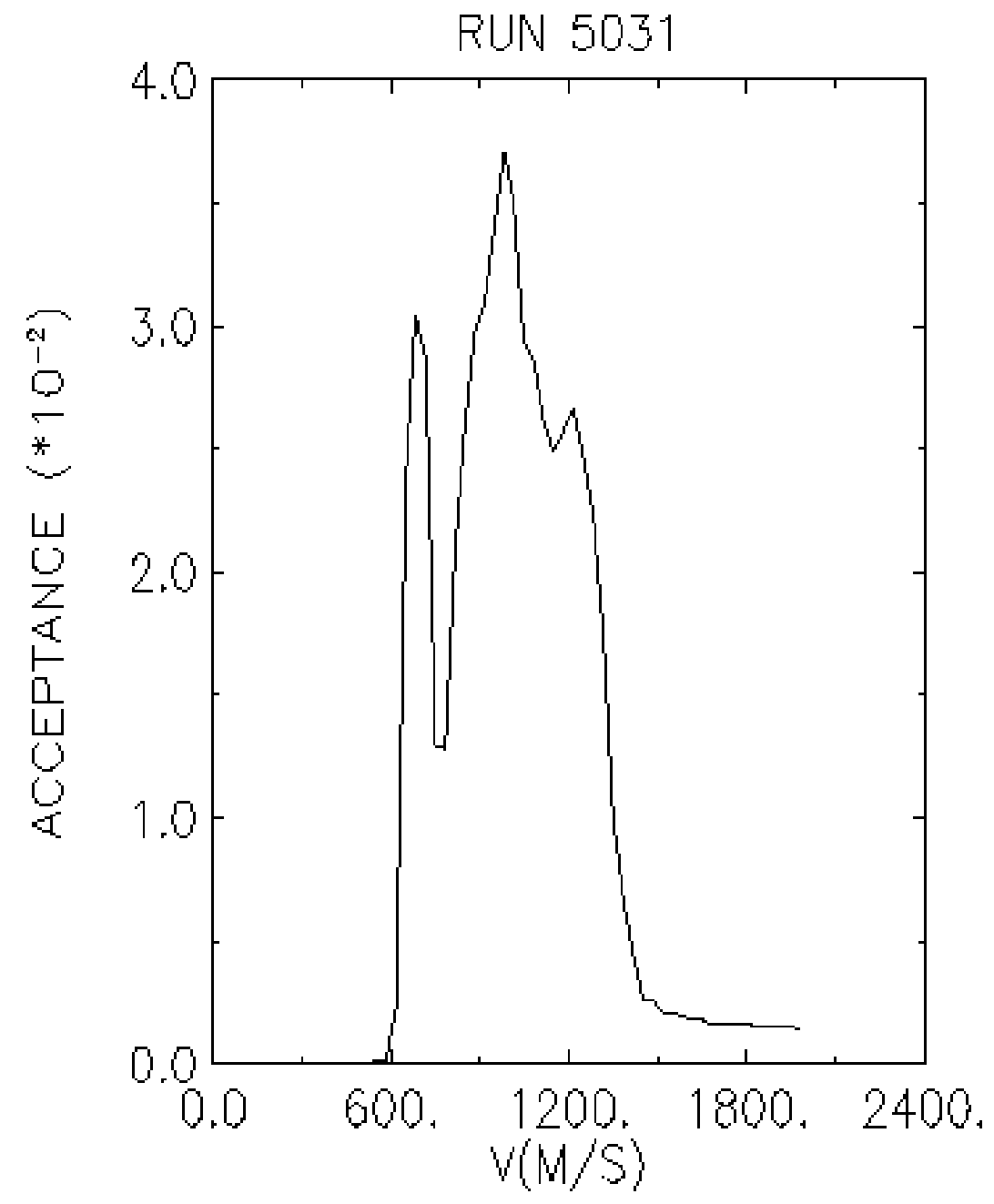

Figure 7: 